\title{
Chapter 12. Hypertension under special conditions
}

\author{
Hypertension Research (2014) 37, 343-348; doi:10.1038/hr.2014.15
}

\section{POINT 12A}

Hypertensive emergencies and urgencies

1. In patients suspected of having a hypertensive emergency, the diagnosis and evaluation of the pathological condition must be made by prompt examination, and treatment must be initiated without delay (Recommendation grade: B, Consensus, Evidence level: VI).

2. As target organ damage progresses rapidly, patients with complications of hypertensive encephalopathy, acute aortic dissection, hypertensive left ventricular failure with pulmonary edema, acute coronary syndrome with severe hypertension, pheochromocytoma crisis and pregnancy with eclampsia or with severe hypertension must be admitted, and intravenous (i.v.) antihypertensive treatment must be started immediately. In principle, treatment should be conducted in facilities with specialists for associated organs such as cardiologists, nephrologists and neurologists, and by hypertension specialists. The same approach as for hypertensive emergency would be taken for accelerated malignant hypertension (Recommendation grade: $\mathbf{B}$, Consensus, Evidence level: VI).

3. Sustained marked hypertension (usually $\geqslant 180 / 120 \mathrm{~mm} \mathrm{Hg}$ ) not accompanied by the progression of acute organ damage or in which acute organ damage may not progress is regarded as a hypertensive urgency. The control of blood pressure can be attempted by oral medication for hypertensive urgency. However, as many such patients have considerable damage in several organs or show resistance to antihypertensive treatment, consultation with a hypertension specialist should be considered (Recommendation grade: B, Consensus, Evidence level: VI).

\section{DIAGNOSIS AND TREATMENT OF HYPERTENSIVE EMERGENCIES AND URGENCIES}

1) Definition, classification and outline

A hypertensive emergency is not simply a condition in which the blood pressure level in patients with high blood pressure is abnormally high but that in which acute damage of target organs such as the brain, heart, kidney and large vessels progresses owing to marked hypertension (usually $\geqslant 180 / 120 \mathrm{~mm} \mathrm{Hg}$ ). This condition must be diagnosed promptly, and antihypertensive treatment must be started immediately.

Hypertensive emergencies include hypertensive encephalopathy, hypertension complicated by acute aortic dissection, hypertensive left ventricular failure accompanied by pulmonary edema, acute coronary syndrome (acute myocardial infarction, unstable angina) accompanied by marked hypertension, pheochromocytoma crisis and pregnancy with eclampsia or with severe hypertension (Table 12-1). ${ }^{958,959}$ In principle, treatment should be carried out in facilities with specialists for associated organs, such as cardiologists, nephrologists and neurologists, and hypertension specialists. Since the publication of the JNC7 Guidelines, ${ }^{113}$ hypertensive emergencies have become well recognized in the United States. The number of patients who were admitted with this condition has increased, whereas the mortality rate has significantly decreased. ${ }^{960}$

Marked hypertension with no rapid progression of organ damage is regarded as a hypertensive urgency. In these cases, there is no evidence of improvement in outcome with emergency antihypertensive treatment. Whether or not the condition is an emergency should not be judged solely on the basis of blood pressure levels. Emergency antihypertensive treatment is not indicated even with an abnormally high blood pressure if there is no acute or progressive organ damage. However, in patients with hypertensive encephalopathy accompanied by acute glomerulonephritis, eclampsia or aortic dissection, emergency treatment is often necessary even when blood pressure is not abnormally high. This pathological condition must be clarified promptly (Table 12-2), the judgment of whether or not it is an emergency case must be made, and which drugs should be used, how they should be administered, what the target blood pressure level should be and how soon it can be attained must be determined. The initiation of treatment for emergencies must not be delayed by spending time on a detailed evaluation.

2) Principles for treatment

Hypertensive emergencies must, in principle, be treated by hospitalization. I.v. antihypertensive treatment should be performed in an intensive care unit or in a similar environment. Invasive monitoring of blood pressure is desirable. Monitoring with a noninvasive procedure for many hours is stressful because of cuff compression by high pressure. Owing to the presence of organ damage and vascular abnormalities, an unnecessarily rapid and excessive decrease in blood pressure is likely to cause ischemic events, such as brain infarction, cortical amaurosis, myocardial infarction and progression of renal dysfunction because of a decrease in organ perfusion pressure. Therefore, the use of drugs and administration methods that allow the degree and rate of decrease in blood pressure to be predicted and the effects to be promptly adjusted is desirable. General targets of blood pressure control are a decrease in mean blood pressure of no more than $25 \%$ during the first $1 \mathrm{~h}$ and to a level of $160 / 100-110 \mathrm{~mm} \mathrm{Hg}$ within the next $2-6$ h. ${ }^{113}$ However, in patients with aortic dissection, 
Table 12-1 Hypertensive emergencies

Accelerated-malignant hypertension with papilledema
Hypertensive encephalopathy
Severe hypertension associated with acute organ damage
Atherothrombotic brain infarction
Brain hemorrhage
Subarachnoid hemorrhage
Head trauma
Acute aortic dissection
Acute left ventricular failure
Acute coronary syndrome
Acute or rapidly progressive renal failure
Severe hypertension after thrombolytic therapy for brain infarction
Excess circulating catecholamines
Pheochromocytoma crisis
Interactions of monoamine oxidase inhibitors with foods or drugs
Use of sympathomimetic drugs
Rebound hypertension after sudden cessation of antihypertensive drugs
Automatic hyperreflexia after spinal cord injury

Pregnant women with a systolic blood pressure of $\geqslant 180 \mathrm{~mm} \mathrm{Hg}$ or a diastolic blood pressure of $\geqslant 120 \mathrm{~mm} \mathrm{Hg}$

Eclampsia

Hypertensive emergencies related to surgery

Severe hypertension in patients requiring emergency surgery

Postoperative hypertension

\section{Postoperative bleeding from vascular suture lines \\ Hypertension after coronary bypass surgery \\ Severe body burns \\ Severe epistaxis}

Accelerated-malignant hypertension, perioperative hypertension, rebound hypertension, burns and epistaxis are classified as urgencies if they are not severe. 'Severe hypertension' here is not in agreement with hypertension categories of the JSH 2014 Guidelines. The blood pressure requiring emergency antihypertensive treatment should be evaluated in each condition. Prepared on the basis of references. 958 and 959.

acute coronary syndrome or hypertensive encephalopathy with no preceding hypertension (acute glomerulonephritis and eclampsia), a blood pressure level at which treatment should be initiated, as well as the target control level, should be set lower.

There is no evidence regarding whether or not antihypertensive therapy prevents eclampsia in pregnant women with a marked increase in blood pressure, but antihypertensive treatment should be started under a diagnosis of hypertensive emergency if systolic blood pressure is $\geqslant 180 \mathrm{~mm} \mathrm{Hg}$ or diastolic blood pressure is $\geqslant 120 \mathrm{~mm} \mathrm{Hg}$, regardless of the presence or absence of convulsion. ${ }^{961}$

Once the initial target of blood pressure control has been reached and oral medication has been started, i.v. treatment is gradually reduced until discontinuation. Only several parenteral drugs for antihypertensive treatment are available in Japan; they are shown in Table 12-3, with their dosage/regimens, time needed to act and duration of actions, adverse effects, areas requiring caution and special indications. With nitroprusside, the rate and degree of decrease in blood pressure are easy to adjust because of the rapid onset and short duration of its action. Cyanate poisoning is unlikely to occur at doses $<2 \mu \mathrm{g} \mathrm{kg}^{-1} \mathrm{~min}^{-1}$. In Japan, however, because of the lack of experience with the use of nitroprusside and its adverse effects, Ca channel blockers have been used more frequently. Caution is necessary in dose adjustment with Ca channel blockers because of their relatively long duration of effect.
In patients with hypertensive urgencies, a long history of hypertension and chronic organ damage is often observed. This suggests that the lower limit of autoregulation of organ blood flow is high. Although antihypertensive treatment should be started within a few hours after diagnosis, blood pressure should be reduced relatively slowly to about $160 / 100 \mathrm{~mm} \mathrm{Hg}$ over $24-48 \mathrm{~h}$ thereafter. Many hypertensive urgencies can be controlled by oral medication. The oral administration of the capsule contents of nifedipine or bolus i.v. injection of the Ca channel blocker, nicardipine, should be avoided, as it can cause an excessive decrease in blood pressure and reflex tachycardia. Ca channel blockers with a relatively rapid onset of action (short-acting or immediate-type Ca channel blockers), angiotensin-converting enzyme (ACE) inhibitors (angiotensin II receptor blockers (ARBs) are also available), the $\alpha \beta$-blocker, labetalol or $\beta$-blockers should be administered orally, or loop diuretics may be used concomitantly depending on the condition. Dose adjustment is easy with captopril because of the rapid onset and relatively short duration of its action, but it should be started at a low dose $(6.25-12.5 \mathrm{mg})$, as it may cause an excessive decrease in blood pressure in patients with malignant hypertension and in a dehydrated state, in which activities of the RA system are enhanced. Caution is necessary in patients with renal dysfunction because hyperkalemia is most likely to occur 1-2 days after commencing treatment with ACE inhibitors. ACE inhibitors should not be used in patients suggested to have bilateral renovascular hypertension or renovascular hypertension with a functionally solitary kidney, as they may cause renal failure. If they are used, monitoring of the serum creatinine and potassium levels is necessary. In patients with hypertensive urgencies, initial treatment is possible at the outpatient clinic, but careful observation in the facility for $5-6 \mathrm{~h}$ after the beginning of administration and on an outpatient basis for 2-3 days thereafter with adjustment of the regimen is necessary. Then, the blood pressure should be controlled to the final target primarily using long-acting antihypertensive drugs, and antihypertensive treatment should be continued. However, treatment by hospitalization is also desirable for a hypertensive urgency in high-risk patients, such as those with a history of cardiovascular disease.

\section{3) Hypertensive encephalopathy}

Hypertensive encephalopathy is a condition in which a rapid or marked increase in blood pressure disrupts the autoregulation of cerebral blood flow, causing brain edema due to unnecessarily high blood flow and pressure. It is most likely to occur when blood pressure increases to $\geqslant 220 / 110 \mathrm{~mm} \mathrm{Hg}$ in chronically hypertensive patients and to $\geqslant 160 / 100 \mathrm{~mm} \mathrm{Hg}$ in normotensive individuals. ${ }^{962}$ Some patients show no proteinuria or hypertensive retinopathy. Hypertensive encephalopathy is the most severe hypertensive emergency leading to brain hemorrhage, disturbance of consciousness, coma and death without appropriate treatment. It is accompanied by exacerbating headache, nausea/vomiting, disturbance of consciousness and convulsions, but focal symptoms are relatively rare. As emergency reduction of blood pressure must, in principle, be avoided in patients with stroke, its exclusion is important. On MRI, vascular edema is often observed in the white matter of the parietal to occipital lobes.

As the autoregulation of cerebral blood flow is disturbed, a rapid and marked decrease in blood pressure is most likely to cause brain ischemia. Treatment should be started with i.v. preparations (continuous i.v. infusion). The rate of decrease in blood pressure should be adjusted by monitoring blood pressure and neurological symptoms. Treatment should be conducted to achieve an $\sim 25 \%$ decrease in 
Table 12-2 Check items for evaluating conditions suspected to be hypertensive emergencies

History, symptoms

History of diagnosis and treatment of hypertension, state of use of sympathomimetic drugs and other drugs, headache, visual impairment, neurological symptoms, nausea/vomiting, chest/back pain, cardiac/respiratory symptoms, oliguria and body weight changes

\section{Physical findings}

Blood pressure: repeat measurements (diastolic pressure is often $\geqslant 120 \mathrm{~mm} \mathrm{Hg}$ ), laterality

Pulse, respiration and body temperature

Evaluation of the body fluid volume: tachycardia, dehydration, edema and measurement of the blood pressure in the standing position

Central nervous system: disturbance of consciousness, convulsion and hemiparesis

Ocular fundus: linear or flame-shaped hemorrhage, soft exudate, retinal edema and papilledema

Neck: jugular vein distension and bruit

Chest: cardiac enlargement, heart murmur, III/IV sounds and moist rales of the lung field

Abdomen: hepatomegaly, bruit and (pulsatile) mass

Limbs: edema and arterial pulsation

Emergency examinations

Urinalysis and blood cell count (including smears)

Blood chemistry (urea nitrogen, creatinine, electrolytes, glucose, LDH and CPK)

ECG, chest X-ray (two directions), arterial blood gas analysis as indicated

Cardiac and abdominal ultrasonography, brain CT scan or MRI and chest/ abdominal CT scan, as indicated

Blood sampling for the measurement of the plasma renin activity, aldosterone, catecholamine and BNP concentrations, as indicated

Abbreviations: CPK, creatin phosphokinase; CT, computed tomography; LDH, low-density lipoprotein.

blood pressure over the first 2-3h. The i.v. injection of nicardipine does not decrease brain tissue oxygen supply, and is useful for treating hypertensive emergencies with neurological signs. ${ }^{963}$ Diltiazem and nitroprusside can also be used. If an increase in extracellular fluid is observed, or resistance has developed, furosemide should be used concomitantly. Hydralazine, which increases the intracranial pressure, must be avoided.

\section{4) Cerebrovascular disease}

See Section 1 of Chapter 6, CEREBROVASCULAR DISEASE.

\section{5) Hypertensive acute left ventricular failure}

Treatment for hypertensive left ventricular failure accompanied by pulmonary edema must be started immediately. The i.v. injection of nitroprusside, which has a rapid onset of action and alleviates not only afterload but also preload by dilating the venous system, is desirable.

On the other hand, the continuous i.v. infusion of nicardipine is also useful, and is routinely used in Japan. Although nitroglycerin has a relatively weak antihypertensive effect, it is useful when hypertensive acute left ventricular failure is concurrent with coronary artery disease. When the body fluid volume is large, carperitide ( $\alpha$-type human atrial natriuretic peptide preparation), ${ }^{964,965}$ which exhibits both vasodilative and diuretic actions, or furosemide should be used with these drugs.

Although no clear target of blood pressure control has been set, blood pressure should be reduced (usually $\sim 10-15 \%$ decrease in systolic blood pressure) by examining the symptomatic improvements. After blood pressure has been reduced to a certain level, treatment should be shifted to oral medication primarily using RA system inhibitors, such as ACE inhibitors and ARBs, combined with Ca channel blockers.

\section{6) Severe hypertension complicating acute coronary syndrome} (acute myocardial infarction and unstable angina)

To treat anginal attacks accompanied by an increase in blood pressure, a nitrate preparation should be administered sublingually or sprayed intra-orally first. If hypertension complicates acute coronary syndrome, nitroglycerin should be administered by continuous i.v. infusion to reduce the myocardial oxygen demand and increase the coronary blood flow as well as lower blood pressure. However, if the concomitant development of right ventricular infarction is suspected due to inferior wall infarction, administration should be avoided. A $\beta$-blocker can be used concomitantly if there is no contraindication such as marked bradycardia. If a $\beta$-blocker cannot be used, or blood pressure cannot be reduced sufficiently, diltiazem should be used. For blood pressure control, a target systolic blood pressure should be $<140 \mathrm{~mm} \mathrm{Hg} .{ }^{966}$ In patients with myocardial infarction, the administration of $\beta$-blockers in the acute stage and ACE inhibitors from the early stage is reported to be useful for improving the prognosis.

\section{7) Aortic dissection}

See Section 4 of Chapter 6, VASCULAR DISEASES.

\section{8) Pheochromocytoma crisis}

Pheochromocytoma crisis refers to a rapid increase in blood pressure related to an excessive secretion of catecholamines. The initial target of blood pressure control is to control a paroxysmal increase in blood pressure. ${ }^{967}$ Phentolamine at $2-5 \mathrm{mg}$ should be intravenously injected at a rate of $1 \mathrm{mg} \mathrm{min}^{-1}$ every $3-5 \mathrm{~min}$ while monitoring the response of blood pressure. After the first dose is injected, phentolamine may be continuously infused via an i.v. route. Oral medication using drugs such as the selective $\alpha$-blocker doxazosin should be started simultaneously. Although $\beta$-blockers are effective for tachycardia, they should be used after the administration of $\alpha$-blockers at a sufficient dose. Monotherapy with $\beta$-blockers enhances $\alpha$-receptor-mediated vasoconstriction, causing an increase in blood pressure; therefore, it is contraindicated. ${ }^{967}$ Pheochromocytoma may cause hypertensive encephalopathy, acute left ventricular failure or accelerated malignant hypertension, and treatment mainly using an $\alpha$-blocker should also be performed in such situations. For intraoperative blood pressure control, the continuous i.v. infusion of phentolamine is sometimes performed in accordance with the patient's blood pressure level. However, the continuous i.v. infusion of nicardipine or nitroglycerin, which less frequently cause side effects, and whose doses can be readily regulated, can also be selected.

\section{9) Accelerated malignant hypertension}

In patients with accelerated malignant hypertension, diastolic blood pressure is $\geqslant 120-130 \mathrm{~mm} \mathrm{Hg}$, and renal dysfunction progresses rapidly if left untreated; the general condition rapidly deteriorates and cardiovascular complications, including heart failure, hypertensive encephalopathy and brain hemorrhage, occur, leading to a poor outcome. Its pathological characteristics are fibrinoid necrosis and proliferative intimitis following arteriolar endothelial damage and the infiltration of plasma components into the vascular wall due to prolonged, marked hypertension; pathological findings in the kidney 
Table 12-3 Parenteral drugs (antihypertensive drugs) for the treatment of hypertensive emergencies

\begin{tabular}{|c|c|c|c|c|c|}
\hline Drug & Dosage/regime & $\begin{array}{l}\text { Onset of } \\
\text { action }\end{array}$ & $\begin{array}{l}\text { Duration } \\
\text { of action }\end{array}$ & Adverse effects/points requiring caution & Special indications \\
\hline \multicolumn{6}{|l|}{ Vasodilators } \\
\hline Nicardipine & $\begin{array}{l}\text { I.v. infusion } \\
0.5-6 \mu \mathrm{g} \mathrm{kg}^{-1} \mathrm{~min}^{-1}\end{array}$ & $5-10 \mathrm{~min}$ & $60 \mathrm{~min}$ & $\begin{array}{l}\text { Tachycardia, headache, flushing and local } \\
\text { phlebitis }\end{array}$ & $\begin{array}{l}\text { Most emergencies. Caution with high } \\
\text { intracranial pressure or acute coronary } \\
\text { syndrome }\end{array}$ \\
\hline Diltiazem & $\begin{array}{l}\text { I.v. infusion } \\
5-15 \mu \mathrm{kg}^{-1} \mathrm{~min}^{-1}\end{array}$ & Within 5 min & $30 \mathrm{~min}$ & $\begin{array}{l}\text { Bradycardia, AV block and sinus arrest. } \\
\text { A low dose in unstable angina }\end{array}$ & Most emergencies except acute heart failure \\
\hline Nitroglycerin & $\begin{array}{l}\text { I.v. infusion } \\
5-100 \mu g \mathrm{~kg}^{-1} \mathrm{~min}^{-1}\end{array}$ & $2-5 \min$ & $5-10 \min$ & $\begin{array}{l}\text { Headache, vomiting, tachycardia, } \\
\text { methemoglobinemia and tolerance with } \\
\text { prolonged use. This drug must be protected } \\
\text { from light }\end{array}$ & Acute coronary syndrome \\
\hline $\begin{array}{l}\text { Sodium } \\
\text { nitroprusside }\end{array}$ & $\begin{array}{l}\text { I.v. infusion } \\
0.25-2 \mu \mathrm{gg}^{-1} \mathrm{~min}^{-1}\end{array}$ & Immediate & $1-2 \mathrm{~min}$ & $\begin{array}{l}\text { Nausea, vomiting, tachycardia and cyanate } \\
\text { poisoning at a high concentration or related to } \\
\text { prolonged administration. This drug must be } \\
\text { protected from light }\end{array}$ & $\begin{array}{l}\text { Most emergencies. Caution with high } \\
\text { intracranial pressure or renal dysfunction }\end{array}$ \\
\hline Hydralazine & $\begin{array}{l}\text { I.v. injection } \\
10-20 \mathrm{mg}\end{array}$ & $10-20 \mathrm{~min}$ & $3-6 h$ & $\begin{array}{l}\text { Tachycardia, flushing, headache, exacerbation } \\
\text { of angina pectoris and sustained hypotension }\end{array}$ & Eclampsia (not the first choice) \\
\hline \multicolumn{6}{|c|}{ Sympatholytic drugs } \\
\hline Phentolamine & $\begin{array}{l}\text { I.v. injection, } 1-10 \mathrm{mg} \\
\text { infusion at } 0.5-2 \mathrm{mg}^{-1} \mathrm{~min}^{-1} \\
\text { after an initial bolus injection } \\
\text { is also possible }\end{array}$ & $1-2 \min$ & $3-10 \mathrm{~min}$ & Tachycardia and headache & $\begin{array}{l}\text { Pheochromocytoma and excess } \\
\text { circulating catecholamines }\end{array}$ \\
\hline Propranolol & $\begin{array}{l}\text { I.v. injection } \\
2-10 \mathrm{mg}\left(1 \mathrm{mg} \mathrm{min}^{-1}\right) \rightarrow \\
2-4 \mathrm{mg} \text { every } 4-6 \mathrm{~h}\end{array}$ & & & Bradycardia, AV block and heart failure & Tachycardia induced by other drugs \\
\hline
\end{tabular}

If pulmonary edema, heart failure or body fluid retention is noted, furosemide or carperitide should be used concomitantly.

are called malignant nephrosclerosis. In this condition, a vicious cycle of progressive renal dysfunction and an increase in blood pressure is established. Ophthalmoscopic findings include retinal hemorrhage, soft exudates, retinal edema and/or papilledema. In the brain, the autoregulation of blood flow is disrupted by vascular damage, and if brain edema occurs hypertensive encephalopathy may result. When there was no effective antihypertensive treatment, the outcome of this pathological condition was as poor as that of malignant neoplasm; hence, it was called malignant hypertension. Malignant hypertension accompanied by papilledema (grade IV according to the KeithWagener classification) and accelerated hypertension accompanied by retinal hemorrhage and exudative lesions (grade III) used to be distinguished. However, as there is no difference between them in the progression of organ damage or survival rate, they have recently been combined as accelerated malignant hypertension. As it is not an independent disease, it is appropriate to call it malignant-phase hypertension. ${ }^{220}$ High blood pressure at the onset of hypertension, interruption of antihypertensive treatment and long-standing mental/ physical stress are involved in the development of malignant hypertension. ${ }^{968}$

Its incidence has recently decreased because of the spread of antihypertensive treatment and improvements in social and living environments. According to results from a single facility in Japan, ophthalmoscopic findings, left ventricular hypertrophy and organ damage such as renal dysfunction in patients treated between 1984 and 1999 were milder than in those treated between 1971 and 1983. ${ }^{969,970}$ In addition, an international study indicated that the 5-year survival rate had markedly improved from 32.0 to $91.0 \%$ in patients treated between 1997 and 2006 in comparison with those treated before $1977 .{ }^{971}$ Not only essential but also secondary hypertension, such as renal parenchymal or renovascular hypertension, may lead to accelerated malignant hypertension.

Although accelerated malignant hypertension is regarded as an urgency, the condition in which arteriolar lesions progress should be classified as an emergency. ${ }^{220}$ The objective of treatment can often be achieved through oral medication. As many patients have a long history of hypertension, a rapid decrease in blood pressure is associated with the risk of ischemia of important organs. Blood pressure should be reduced to no less than a diastolic pressure of $100-110 \mathrm{~mm} \mathrm{Hg}$ during the first $24 \mathrm{~h} .{ }^{220} \mathrm{ACE}$ inhibitors and ARBs may be effective because body fluid is reduced owing to pressure diuresis, and because hyperactivity of the RA system is closely involved in the pathogenesis in patients with this condition resulting from essential hypertension ${ }^{970}$ or the renal crisis of collagen diseases. However, as these drugs may cause an excessive decrease in blood pressure, their administration should be started at a low dose. If a decrease in the body fluid volume is clear, fluid replacement with physiological saline is necessary. On the other hand, loop diuretics should be used if sodium/water retention is present.

\section{TRANSIENT INCREASES IN BLOOD PRESSURE}

\section{POINT 12B}

1. If a marked temporary increase in blood pressure is not accompanied by progressive organ damage, emergency antihypertensive treatment is unnecessary except in cases of pheochromocytoma.

2. If a marked increase in blood pressure persists, Ca channel blockers with an intermediate duration of action, ACE 
Table 12-4 Conditions that may exhibit marked transient increases in blood pressure

- Impairment of the baroreflex mechanism

- Hyperventilation associated with anxiety

- Panic attacks (panic disorder)

- Pseudopheochromocytoma

- Pheochromocytoma

inhibitors, or ARBs should be orally administered (Recommendation grade: C1, Evidence level: VI).

3. If a sufficient inquiry suggests psychological factors, the patient may be referred to a specialist in mental health care (Recommendation grade: $\mathbf{C} 1$, Evidence level: $\mathbf{V I}$ ).

Marked transient increases in blood pressure, except those caused by pheochromocytoma, do not require emergency antihypertensive treatment unless there is progressive or chronic organ damage (Table 12-4). In patients with impairment of the baroreflex mechanism, such as elderly patients and those with autonomic neuropathy, blood pressure markedly changes, and grade III or severer hypertension is observed in some patients. To such patients, short-acting antihypertensive drugs such as the contents of nifedipine capsules may be administered, but should be avoided as a rapid and excessive decrease in blood pressure may induce ischemia of the main organs such as the brain and heart.

Causes of an increase in blood pressure, such as pain and urinary retention, should be resolved, and, if blood pressure remains high even on repeated measurements, Ca channel blockers with an intermediate duration of action (administered twice a day), ARBs or ACE inhibitors should be orally administered.

Pheochromocytoma is a type of secondary hypertension characterized by physical symptoms such as paroxysmal headache, chest pain, dizziness, nausea, palpitation, flushing and diaphoresis, as well as a marked increase in blood pressure. For the diagnosis and treatment of pheochromocytoma, see Section 3 of Chapter 13, ENDOCRINE HYPERTENSION. On the other hand, it is suggested that mental factors are involved in 'pseudopheochromocytoma', in which there is no tumorous lesion on diagnostic imaging despite clinical symptoms resembling pheochromocytoma, and there are only slight increases in the levels of catecholamines. ${ }^{972}$ Similarly, a paroxysmal increase in blood pressure with symptoms such as tachycardia, palpitation, headache and dyspnea is observed in patients with panic disorder (panic attacks) or hyperventilation. Panic disorder is mutually associated with hypertension and is reported to be a risk factor for cardiovascular diseases such as myocardial infarction and stroke. ${ }^{973,974}$ As antihypertensive drugs, $\alpha \beta$-blockers and centrally acting antihypertensive drugs are appropriate for these patients in whom the involvement of stress or mental factors is marked, but it is difficult to control blood pressure using antihypertensive drugs alone in many cases. The use of antipsychotic drugs, such as antidepressants, through consultations with a specialist, and introduction of psychological approaches such as psychotherapy and behavioral therapy by specialists are necessary. ${ }^{975}$

\section{PREOPERATIVE AND POSTOPERATIVE BLOOD PRESSURE MANAGEMENT}

\section{POINT 12C}

1. For the prevention of perioperative complications in hypertensive patients, a differential diagnosis of secondary hypertension, such as pheochromocytoma, and evaluation of hypertensive organ damage/complications are important.

2. If blood pressure is $\geqslant 180 / 110 \mathrm{~mm} \mathrm{Hg}$ at the time of elective surgery, blood pressure control should be predominantly performed (Recommendation grade: $\mathrm{C} 1$, Evidence level: VI).

3. As a rule, blood pressure should be controlled by continuous oral or i.v. antihypertensive treatment throughout the perioperative period, including the administration on the morning of surgery (Recommendation grade: C1, Evidence level: VI).

4. In high-risk patients with coronary artery disease, the use of $\beta$-blockers should be considered (Recommendation grade: $B$, Consensus).

5. In patients taking diuretics, ARBs or ACE inhibitors, much attention should be paid to intra-/postoperative hypotension, a decrease in the body fluid volume and renal dysfunction.

6. Elimination of pain/anxiety and excitation is also important for controlling the increase in blood pressure (Recommendation grade: $\mathrm{C} 1$, Evidence level: VI).

\section{1) Preoperative evaluation of hypertension}

Elective surgery is a good opportunity to assess hypertension and evaluate the therapeutic approach. In patients with untreated hypertension, perioperative risk assessment through the evaluation of hypertensive organ damage and complications, such as those of the brain, heart, kidney, blood vessels and fundus of the eye, as well as a differential diagnosis of secondary hypertension, is important. In particular, it is necessary to examine the presence or absence of conditions in which ischemic complications due to perioperative blood pressure decreases are most likely to occur, such as cerebrovascular disease, carotid artery stenosis, left ventricular hypertrophy, coronary artery disease and renal dysfunction. If there is a risk of ischemic complications, consistent blood pressure management from the preoperative period is necessary to avoid excessive perioperative changes in blood pressure.

In patients suspected of having pheochromocytoma, examinations should be performed by postponing surgery, and, if a definitive diagnosis is made, the tumor must be removed before the intended surgery. Conditions such as renovascular hypertension, primary aldosteronism and Cushing's syndrome pose few problems if blood pressure is controlled to the grade I hypertension or lower level before surgery, but manageable secondary hypertension should be treated before elective surgery.

Grade I or II hypertension is not an independent risk factor for perioperative cardiovascular complications, but if blood pressure is $\geqslant 180 / 110 \mathrm{~mm} \mathrm{Hg}$ before elective surgery blood pressure control should be predominantly performed, considering the risk of postponing surgery. ${ }^{976}$ When conducting endoscopic surgery or invasive examinations in patients with grade III hypertension or high-risk patients, the applicability of those procedures must be judged individually by evaluating their risks and merits.

2) Use of antihypertensive drugs in the perioperative period Antihypertensive drugs should be administered until the day of surgery, in principle, and be resumed as soon as possible after surgery. In particular, if $\beta$-blockers are used, caution is necessary not to interrupt treatment considering the risk of increases in the heart rate and blood pressure. Although there is no evidence regarding whether or not the perioperative use of $\beta$-blockers improves the prognosis, ${ }^{977} \beta$-blockers may protect against perioperative stress and sympathetic hyperactivity, reducing the risks of ischemic cardiac 
complications and atrial fibrillation. ${ }^{976}$ Therefore, in high-risk patients with coronary artery disease, the start of $\beta$-blockers should be considered.

If there is a risk of intraoperative hypotension, postoperative dehydration or hypokalemia, the preoperative discontinuation of diuretics must also be considered. If the patient is being treated with an ACE inhibitor or an ARB, it may induce a decrease in blood pressure or renal dysfunction associated with a perioperative decrease in the body fluid volume; 978 therefore, some reports recommend the preoperative discontinuation of administration. ${ }^{976}$ In particular, whether or not the preoperative discontinuation of administration is possible should be individually examined in highrisk patients including elderly individuals, considering the condition and surgical invasiveness.

Increases in blood pressure during an emergency or elective surgery should be controlled by the continuous i.v. infusion of Ca channel blockers (nicardipine and diltiazem), nitroglycerin or nitroprusside. As hemodynamics remain unstable after surgery, antihypertensive treatment should be started as early as possible, intravenously if oral administration is impossible. Appropriate treatment is also necessary for factors that increase blood pressure, such as postoperative pain, anxiety and excitation. Administration of the contents of nifedipine capsules must be avoided because the degree or rate of blood pressure reduction cannot be controlled.

\section{3) Dental surgery and blood pressure management}

As cardiovascular disease such as stroke may also occur during dental treatment, evaluation of the presence or absence of hypertension and the state of blood pressure is also necessary before dental treatment. If blood pressure is $\geqslant 180 / 110 \mathrm{~mm} \mathrm{Hg}$, medical consultation and referral to an internist should precede dental treatment except for emergency procedures. ${ }^{979}$ Patients receiving antihypertensive medication should be advised to take their medication on the day of dental treatment. Dental procedures that involve pain or anxiety or require a prolonged time induce an increase in blood pressure. ${ }^{980}$ As local anesthetics containing adrenaline (epinephrine) slightly increase blood pressure, their doses should be carefully determined while ensuring that there is sufficient anesthesia for pain control. ${ }^{979,980}$ Prescription of a tranquilizer can be considered in patients complaining of intense anxiety.

\section{Citation Information}

We recommend that any citations to information in the Guidelines are presented in the following format:

The Japanese Society of Hypertension Guidelines for the Management of Hypertension (JSH 2014). Hypertens Res 2014; 37: 253-392.

Please refer to the title page for the full list of authors. 